\title{
Ghost Dark Energy in the DGP Braneworld
}

\author{
M. Abdollahi Zadeh ${ }^{1}$ and A. Sheykhi $\mathbb{D D}^{1,2}$ \\ ${ }^{1}$ Physics Department and Biruni Observatory, College of Sciences, Shiraz University, Shiraz 71454, Iran \\ ${ }^{2}$ Research Institute for Astronomy and Astrophysics of Maragha (RIAAM), P.O. Box 55134-441, Maragha, Iran
}

Correspondence should be addressed to A. Sheykhi; asheykhi@shirazu.ac.ir

Received 8 July 2018; Accepted 20 September 2018; Published 14 October 2018

Academic Editor: Ricardo G. Felipe

Copyright (c) 2018 M. Abdollahi Zadeh and A. Sheykhi. This is an open access article distributed under the Creative Commons Attribution License, which permits unrestricted use, distribution, and reproduction in any medium, provided the original work is properly cited. The publication of this article was funded by SCOAP ${ }^{3}$.

\begin{abstract}
We investigate the ghost model of dark energy in the framework of DGP braneworld. We explore the cosmological consequences of this model by determining the equation of state parameter, $\omega_{D}$, the deceleration, and the density parameters. We also examine the stability of this model by studying the squared of the sound speed in the presence/absence of interaction term between dark energy and dark matter. We find out that in the absence of interaction between two dark sectors of the universe we have $\omega_{D} \longrightarrow-1$ in the late time, while in the presence of interaction $\omega_{D}$ can cross the phantom line -1 . In both cases the squared of sound speed $v_{s}^{2}$ does not show any signal of stability. We also determine the statefinder diagnosis of this model as well as the $\omega_{D}-\omega_{D}^{\prime}$ plane and compare the results with the $\Lambda \mathrm{CDM}$ model. We find that $\omega_{D}-\omega_{D}^{\prime}$ plane meets the freezing region in the absence of interaction between two dark sectors, while it meets both the thawing and the freezing regions in the interacting case.
\end{abstract}

\section{Introduction}

The current acceleration of the universe expansion is strongly confirmed by the type Ia supernova observations [1] and also supported by the astrophysical data from WMAP [2]. A component which is responsible for this acceleration is called dark energy (DE) with negative pressure, which can overcome the gravity force between the galaxies and push them to accelerate, though its nature and origin is still an open question in the modern cosmology. There are two approaches for explanation of the cosmic acceleration. The first one is the modified gravity models such as $f(R)$ gravity [3] and scalartensor theories [4], and the second approach is the idea of the existence of a strange type of energy whose gravity is repulsive such as the cosmological constant $\Lambda$ [5] and the dynamical DE models [6,7]. Against the cosmological constant $\Lambda$ which has constant equation of state (EoS) parameter, $\omega_{D}=-1$, further observations detect a small variation in the EoS parameter of DE in favor of a dynamical DE with $\omega_{D}>-1$ in the past and even $\omega_{D}<-1$ in the late time [8].

An interesting model for probing the dynamical DE model is the ghost dark energy (GDE) model proposed in [9]. The advantages of this model are that it does not introduce any new degree of freedom in contrast to the most DE models which explain the accelerated expansion by introducing new degree(s) of freedom or by modifying the underlying theory of gravity. This is important because, with introducing new degrees of freedom, one needs to investigate the nature and new consequences in the universe so it seems to be impressive and economic if we can explain DE puzzle by using currently known fluids and fields of nature. Actually, GDE model which is based on the Veneziano ghost in Quantum Chromodynamics (QCD) can act as the source of $\mathrm{DE}[10]$ and its existence are required for resolution of the $\mathrm{U}(1)$ problem in QCD [11]. Indeed, the ghosts are decoupled from the physical states and make no contribution in flat Minkowski space, but it produces a small vacuum energy density in a dynamic background or a curved spacetime proportional to $\Lambda_{\mathrm{QCD}}^{3} H$, where $H$ is the Hubble parameter and $\Lambda_{\mathrm{QCD}}$ is QCD mass scale of order a $100 \mathrm{MeV}$ [12]. Different features of GDE have been studied in ample details [13].

Independent of the DE puzzle, for explanation of the cosmic acceleration, special attention is also paid to extra dimensional theories, in which our universe is realized as a 3-brane embedded in a higher dimensional spacetime. Based 
on the braneworld model, all the particle fields in the standard model are confined to a four-dimensional brane, while gravity is free to propagate in all dimension. One of the original models of braneworld is introduced by Dvali-GabadadzePorrati (DGP) [14], which describes our universe as a $4 \mathrm{D}$ brane embedded in a 5D Minkowskian bulk with infinite size. In this model the recovery of the usual gravitational laws on the brane is obtained by adding an Einstein-Hilbert term to the action of the brane computed with the brane intrinsic curvature. It is a well-known that the DGP model has two branches of solutions. The self-accelerating branch of DGP model can explain the late time cosmic speed-up without recourse to DE or other components of energy [15, 16]. However, the self-accelerating DGP branch has ghost instabilities and it cannot realize phantom divide crossing by itself. To realize phantom divide crossing it is necessary to add at least a component of energy on the brane. On the other hand, the normal DGP branch cannot explain acceleration but it has the potential to realize a phantomlike phase by dynamical screening on the brane. Adding a DE component to the normal branch solution brings new facilities to explain late time acceleration and also better matching with observations. These are the motivations to add $\mathrm{DE}$ to this braneworld setup $[17,18]$. In this work we would like to investigate the GDE model in the framework of the DGP braneworld. This study is of great importance, since we can incorporate and disclose the effects of the extra dimension on the evolution of the cosmological parameters on the brane when the DE source is in the form of GDE.

This paper is organized as follows. In Section 2 we formulate the GDE model in the context of the DGP braneworld. We also consider both interacting and noninteracting cases and explore various cosmological parameters as well as cosmological planes. Besides the discussion of instability analysis, we study the $\omega_{D}-\omega_{D}^{\prime}$ plane and properties of statefinder parameters. We finish with closing remarks in Section 3.

\section{The GDE in the DGP Model}

In the DGP cosmology, a homogeneous, spatially flat, and isotropic 3-dimensional brane which is embedded in a 5dimensional Minkowskian bulk can be described by the following Friedmann equation [19]

$$
H^{2}=\left(\sqrt{\frac{\rho_{m}+\rho_{D}}{3 m_{p}^{2}}+\frac{1}{4 r_{c}^{2}}}+\frac{\epsilon}{2 r_{c}}\right)^{2},
$$

or equivalently

$$
H^{2}-\frac{\epsilon}{r_{c}} H=\frac{1}{3 m_{p}^{2}}\left(\rho_{m}+\rho_{D}\right),
$$

where $H=\dot{a} / a$ is the Hubble parameter, $r_{c}=m_{\mathrm{pl}}^{2} /\left(2 m_{5}^{3}\right)$ [20] is the crossover length scale reflecting the competition between $4 \mathrm{D}$ and $5 \mathrm{D}$ effects of gravity, and $\epsilon= \pm 1$ corresponds to the two branches of solutions of the DGP model. Before going any further, it is worth noting that if $H^{-1} \ll r_{c}$ (early times) the $4 \mathrm{D}$ general relativity is recovered; otherwise the $5 \mathrm{D}$ effect becomes significant. Also $\epsilon=+1$ corresponds to the self-accelerating solution where the universe may accelerate in the late time purely due to modification of gravity $[15,16]$, while $\epsilon=-1$ can produce the acceleration only if a DE component is included on the brane. Here, to accommodate GDE into the formalism we take $\epsilon=-1$.

The fractional energy density parameters are defined as

$$
\begin{aligned}
& \Omega_{m}=\frac{\rho_{m}}{3 m_{p}^{2} H^{2}}, \\
& \Omega_{D}=\frac{\rho_{D}}{3 m_{p}^{2} H^{2}}, \\
& \Omega_{r_{c}}=\frac{1}{4 r_{c}^{2} H_{0}^{2}},
\end{aligned}
$$

where $H_{0}$ is the Hubble parameter at redshift $z=0$. The Friedmann equation (2) can be rewritten in terms (3) as

$$
\Omega_{m}+\Omega_{D}+2 \epsilon \frac{H_{0}}{H} \sqrt{\Omega}_{r_{c}}=1
$$

We introduce $\Omega_{D G P}=2 \epsilon \sqrt{\Omega_{r_{c}}} H_{0} / H$, which comes from the extra dimension. Thus the Friedmann equation (4) can be reexpressed as

$$
\Omega_{m}+\Omega_{D}+\Omega_{D G P}=1 .
$$

For the GDE density we have

$$
\rho_{D}=\alpha H
$$

where $\alpha$ is a constant of order $\Lambda_{\mathrm{QCD}}^{3}$ and $\Lambda_{\mathrm{QCD}}$ is the QCD mass scale [12]. Taking the time derivative of the energy density $\rho_{D}$ and using (6) we obtain

$$
\dot{\rho}_{D}=\rho_{D} \frac{\dot{H}}{H}
$$

For the FRW universe filled with DE and DM, with mutual interaction, the energy-momentum conservation law can be written as

$$
\begin{aligned}
\dot{\rho}_{m}+3 H \rho_{m} & =Q, \\
\dot{\rho}_{D}+3 H\left(1+\omega_{D}\right) \rho_{D} & =-Q,
\end{aligned}
$$

where $Q=3 b^{2} H\left(\rho_{D}+\rho_{m}\right)$ is considered as the interaction term between DE and DM also $b^{2}$ is the coupling constant of interaction $Q$.

We know that (i) our universe is in a DE dominated phase and (ii) our universe that is our habitat is stable. These imply that any variable DE model should result in a stable DE dominated universe. So it is worth investigating the stability of the GDE in DGP braneworld against perturbation. The intended indicator for checking the stability of a proposed DE model is to study the behavior of the squared sound speed $\left(v_{s}^{2}=d P / d \rho\right)$ [21]. If $v_{s}^{2}<0$ we have the classical instability of a given perturbation because the perturbation of 
the background energy density is an oscillatory function and may grow or decay with time. When $v_{s}^{2}>0$, we expect a stable universe against perturbations because the perturbation in the energy density propagates in the environment. We continue discussion of stability in the linear perturbation regime where the perturbed energy density of the background can be written as

$$
\rho(t, x)=\rho(t)+\delta \rho(t, x)
$$

where $\rho(t)$ is unperturbed background energy density. For the energy conservation equation $\left(\nabla_{\mu} T^{\mu \nu}=0\right)$ which yields [21]

$$
\delta \ddot{\rho}=v_{s}^{2} \nabla^{2} \delta \rho(t, x)
$$

we encounter two cases. In the first case where $v_{s}^{2}>0$, we observe an ordinary wave equation which has a wave solution in the form $\delta \rho=\delta \rho_{0} e^{-i \omega t+i \vec{k} \cdot \vec{x}}$ (stable universe). In the second case where $v_{s}^{2}<0$, the frequency of the oscillations becomes pure imaginary and the density perturbations will grow with time as $\delta \rho=\delta \rho_{0} e^{\omega t+i \vec{k} \cdot \vec{x}}$ (unstable universe). Since $v_{s}^{2}$ plays a crucial role in determining the stability of DE model, we rewrite it in terms of EoS parameter as

$$
v_{s}^{2}=\frac{\dot{P}}{\dot{\rho}}=\frac{\dot{\rho}_{D} w_{D}+\rho_{D} \dot{w}_{D}}{\dot{\rho}_{D}(1+u)+\rho_{D} \dot{u}},
$$

where $P=P_{D}$ is the pressure of DE, $\rho=\rho_{m}+\rho_{D}$ is the total energy density of DE and DM, and $u=\Omega_{m} / \Omega_{D}$ is the energy density ration.

On the other sides, Sahni et al. [22] proposed new geometrical diagnostic pair parameter $\{r, s\}$, known as statefinder parameter, for checking the viability of newly introduced DE models. Unlike the physical variables which depend on the properties of physical fields describing DE models, the statefinder pair primarily depends on the scale factor and hence it depends on the metric of the spacetime. The $r$ and $s$ parameters are defined as [22]

$$
\begin{aligned}
& r=\frac{\dddot{a}}{a H^{3}}, \\
& s=\frac{r-1}{3(q-1 / 2)},
\end{aligned}
$$

where $r$ can rewrite as

$$
r=1+3 \frac{\dot{H}}{H^{2}}+\frac{\ddot{H}}{H^{3}} .
$$

and then

$$
r=2 q^{2}+q-\frac{\dot{q}}{H}
$$

Let us note that in the $\{r, s\}$ plane, $s>0$ corresponds to a quintessence-like model of DE and $s<0$ corresponds to a phantom-like model of DE. Also the studies on a flat $\Lambda$ CDM model and matter dominated universe (SCDM) show that for these models $\{r, s\}=\{1,0\}$ and $\{r, s\}=\{1,1\}$, respectively. In above equations $q$ is the deceleration parameter which is given by

$$
q=-1-\frac{\dot{H}}{H^{2}}
$$

In what follows we discuss the $\omega_{D}-\omega_{D}^{\prime}$ plane which introduced by Caldwell and Linder [23] for analyzing the dynamical property of various DE models and distinguish these models $\left(\omega_{D}^{\prime}\right.$ represents the evolution of $\left.\omega_{D}\right)$. The models can be categorized into two different classes: (i) $\omega_{D}^{\prime}>0$ and $\omega_{D}<0$ which present the thawing region. (ii) $\omega_{D}^{\prime}<0$ and $\omega_{D}<0$ which present the freezing region. It should be noted that the $\Lambda C D M$ model corresponds to a fixed point $\left\{\omega_{D}=\right.$ $\left.-1, \omega_{D}^{\prime}=0\right\}$ in the $\omega_{D}-\omega_{D}^{\prime}$ plane. We shall consider the noninteracting and interacting cases, separatively. It deserves to mention here that in all figures we selected values for the fixed parameters, which is custom and the observational evidence confirms it, as $\Omega_{r_{c}}=0.0003$ [24] or $\Omega_{D}(z=$ $0)$ that can be between 0.7 and .75 and $H(z=0)$ that can be between 60 and 100 . It should be noted that the results and conclusions are not sensitive to these choices and also do not do any observational work on this title; thus we by helping papers and experience selected these values for the fixed parameters.

2.1. Noninteracting Case. We start to obtain the cosmological parameters for GDE in the DGP braneworld by ignoring the interaction term $(Q=0)$. The deceleration parameter $q$ can be obtained by taking the time derivative of (2), which lead to

$$
\frac{\dot{H}}{H^{2}}=\frac{-3\left(1-\Omega_{D G P}\right)+3 \Omega_{D}}{2-\Omega_{D G P}-\Omega_{D}} .
$$

Using relation (16), we find

$$
q=-1-\frac{-3\left(1-\Omega_{D G P}\right)+3 \Omega_{D}}{2-\Omega_{D G P}-\Omega_{D}} .
$$

Inserting (7) in (9) we have

$$
\omega_{D}=-1-\frac{1}{3} \frac{\dot{H}}{H^{2}}
$$

where by replacing (17) in it, we get

$$
\omega_{D}=-\frac{1}{2-\Omega_{D G P}-\Omega_{D}} .
$$

Also we obtain $\omega_{D}^{\prime}$ from the above equation as

$$
\omega_{D}^{\prime}=-\frac{3\left(-1+\Omega_{D G P}+\Omega_{D}\right)\left(\Omega_{D G P}+\Omega_{D}\right)}{\left(-2+\Omega_{D G P}+\Omega_{D}\right)^{3}} .
$$

Note that, in order to find the evolution of density parameter $\Omega_{D}$, we take the time derivative of relation $\Omega_{D}=$ $\rho_{D} /\left(3 m_{p}^{2} H^{2}\right)$, after combining the result with (7) and (17), which yields

$$
\Omega_{D}^{\prime}=\Omega_{D}(1+q) .
$$




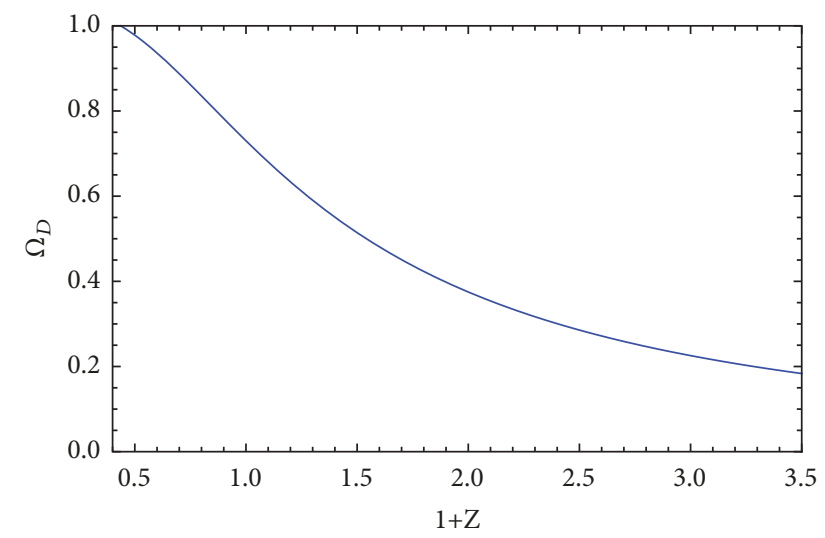

Figure 1: The evolution of $\Omega_{D}$ versus redshift parameter $z$ for noninteracting GDE in DGP model. Here, we have taken $\Omega_{D}(z=$ $0)=0.73, H(z=0)=67$, and $\Omega_{r_{c}}=0.0003$.

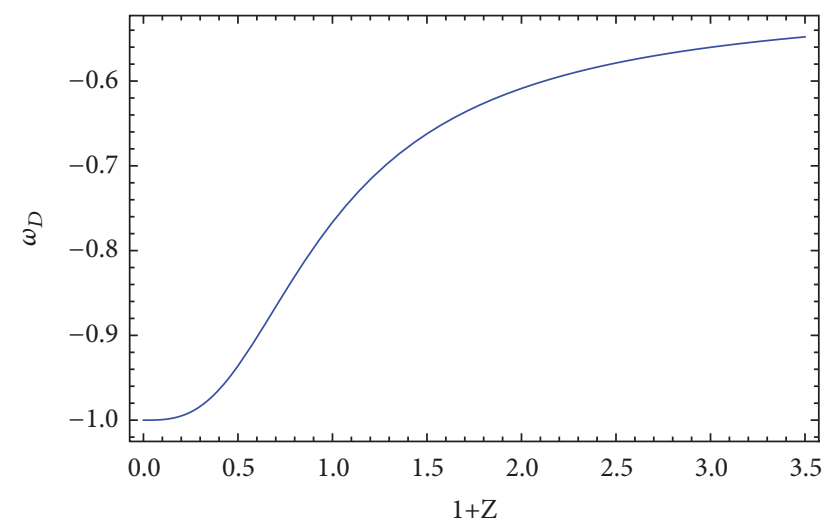

Figure 2: The evolution of $\omega_{D}$ versus redshift parameter $z$ for noninteracting GDE in DGP model. Here, we have taken $\Omega_{D}(z=$ $0)=0.73, H(z=0)=67$, and $\Omega_{r_{c}}=0.0003$.

In Figure 1, we plot the evolution of $\Omega_{D}$ versus redshift parameter $z$. It is obvious that $\Omega_{D}$ tends to 0 in the early universe where $1+z \longrightarrow \infty$, while at the late time where $1+z \longrightarrow 0$, we have $\Omega_{D} \longrightarrow 1$. Clearly, (20) for the EoS parameter shows that, at the late time where $\Omega_{D} \longrightarrow 1$, the EoS parameter mimics the cosmological constant, namely, $\omega_{D} \longrightarrow-1$, which is showed in Figure 2. In Figure 3, the behavior of the deceleration parameter $q$ is plotted and indicates that indeed there is a decelerated expansion at the early stage of the universe followed by an accelerated expansion. The energy density ratio is defined as $u=\Omega_{m} / \Omega_{D}$, which by using (5) can be written

$$
u=-1+\frac{1}{\Omega_{D}}\left(1-\Omega_{D G P}\right) .
$$

Differentiating (23) and (20) and then substituting the results in (12) we get the squared of sound speed as

$$
v_{s}^{2}=-\frac{2 \Omega_{D}\left(-1+\Omega_{D G P}+\Omega_{D}\right)}{\left(-2+\Omega_{D G P}\right)\left(-2+\Omega_{D G P}+\Omega_{D}\right)^{2}} .
$$

The evolution of $v_{s}^{2}$ against $z$ for the noninteracting GDE in the framework of DGP braneworld is plotted in Figure 4. From graphical analysis of $v_{s}^{2}$ one concludes that this model does not indicate any signal of stability, that is, $v_{s}^{2}<0$ during the history of the universe. We can also find the statefinder parameters $r$ and $s$ by taking derivative of (18) and using (13) and (15). The results are

$$
\begin{aligned}
r= & 10+\frac{18}{\left(-2+\Omega_{D G P}+\Omega_{D}\right)^{3}}+\frac{45}{\left(-2+\Omega_{D G P}+\Omega_{D}\right)^{2}} \\
& +\frac{36}{-2+\Omega_{D G P}+\Omega_{D}}, \\
s= & \frac{2\left(-1+\Omega_{D G P}+\Omega_{D}\right)^{2}}{\left(-2+\Omega_{D G P}+\Omega_{D}\right)^{2}} .
\end{aligned}
$$

The graphical behavior of the statefinder parameters $\{r, s\}$ given in (25) and (26) is plotted in Figures 5 and 6, showing that, at late time where $\Omega_{D} \longrightarrow 1$, we have $\{r, s\}=\{1,0\}$ which implies that GDE mimics the cosmological constant at the late time, as expected.

Let us study the trajectory in the statefinder plane and analyze this model from the statefinder viewpoint. For this purpose, we plot the statefinder diagram in the $r-s$ in Figure 7, which shows the cure gets to the point $\{r, s\}=\{1,0\}$ in the end, which implies that the model corresponds to the $\Lambda \mathrm{CDM}$ model at the late time. For complementarity of the diagnostic, we also plot the trajectories of statefinder pair $r-q$ in Figure 8 which ends in the future to $r=1, q=-1$ corresponding to the de Sitter expansion.

The $\omega_{D}-\omega_{D}^{\prime}$ plane for the noninteracting GDE in the DGP scenario is shown in Figure 9. Again, we see that this plane corresponds to $\Lambda \mathrm{CDM}$ model, i.e., $\left(\omega_{D}=-1, \omega_{D}^{\prime}=0\right)$, and the trajectory meets the freezing region as well.

2.2. Interacting Case. Differentiating the modified Friedmann equation (2) and using (7) and (8) we reach

$$
\begin{aligned}
\frac{\dot{H}}{H^{2}} & =\frac{3\left(b^{2}-1\right)\left(1-\Omega_{D G P}\right)+3 \Omega_{D}}{2-\Omega_{D G P}-\Omega_{D}}, \\
q & =-1-\frac{3\left(b^{2}-1\right)\left(1-\Omega_{D G P}\right)+3 \Omega_{D}}{2-\Omega_{D G P}-\Omega_{D}} .
\end{aligned}
$$

Next, the EoS parameter can be determined by substituting (7) into the semiconservation law equation (9) and using (27). We find

$$
\omega_{D}=\frac{b^{2}\left(\Omega_{D G P}-2\right)\left(\Omega_{D G P}-1\right)+\Omega_{D}}{\Omega_{D}\left(-2+\Omega_{D G P}+\Omega_{D}\right)} .
$$

Taking differentiation with respect to $x=\ln a$ from above equation we get 


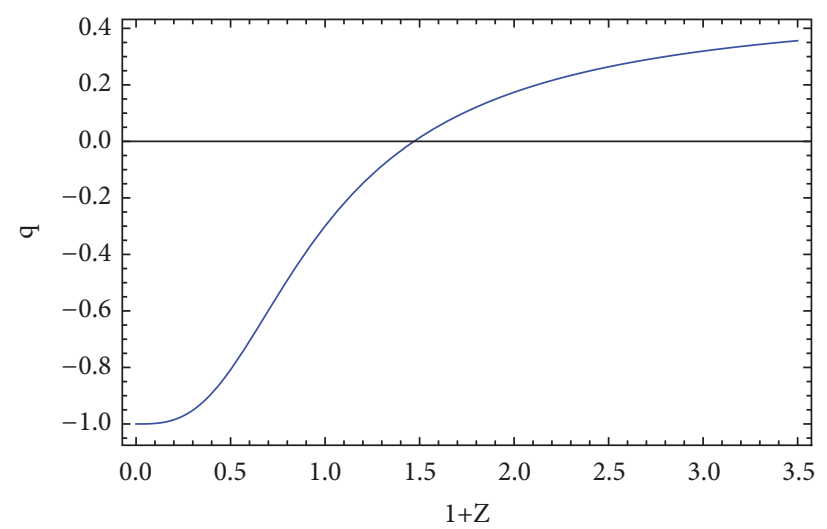

FIGURE 3: The evolution of the deceleration parameter $q$ versus redshift parameter $z$ for noninteracting GDE in DGP model. Here, we have taken $\Omega_{D}(z=0)=0.73, H(z=0)=67$, and $\Omega_{r_{c}}=0.0003$.

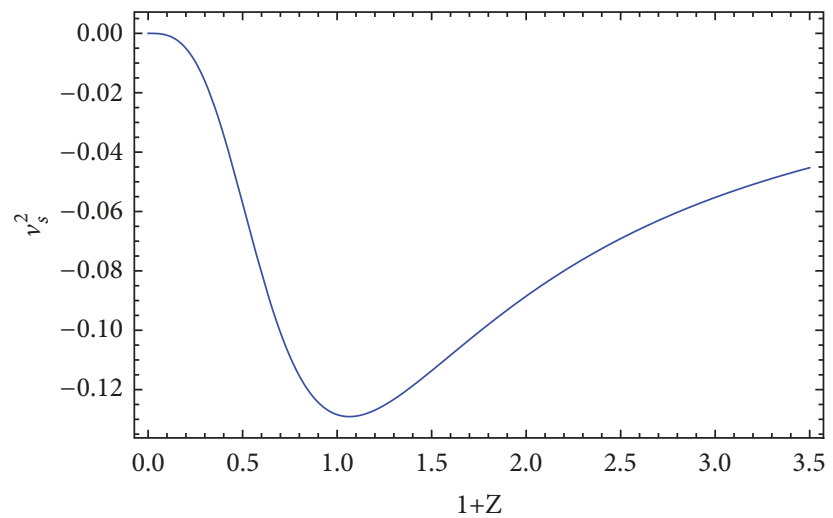

FIGURE 4: The evolution of the squared of sound speed $v_{s}^{2}$ versus redshift parameter $z$ for noninteracting GDE in DGP model. Here, we have taken $\Omega_{D}(z=0)=0.73, H(z=0)=67$, and $\Omega_{r_{c}}=0.0003$.

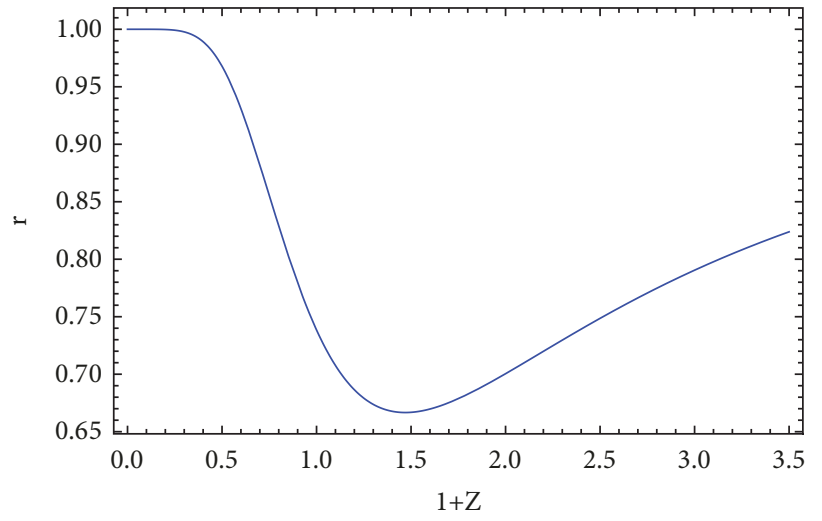

FIGURE 5: The evolution of the statefinder parameter $r$ versus the redshift parameter $z$ for noninteracting GDE in DGP model. Here, we have taken $\Omega_{D}(z=0)=0.73, H(z=0)=67$, and $\Omega_{r_{c}}=0.0003$.

$$
\omega_{D}^{\prime}=-\frac{3\left[\left(-1+b^{2}\right)\left(-1+\Omega_{D G P}\right)-\Omega_{D}\right]\left[b^{2}\left(-2+\Omega_{D G P}\right)^{2}+\left(-\Omega_{D G P}+b^{2}\left(-4+3 \Omega_{D G P}\right)-\Omega_{D}\right) \Omega_{D}\right]}{\Omega_{D}\left(-2+\Omega_{D G P}+\Omega_{D}\right)^{3}},
$$

where the prime indicates derivative with respect to $x=\ln a$. We can obtain the equation of motion for $\Omega_{D}$ as

$$
\Omega_{D}^{\prime}=\frac{3 \Omega_{D}\left(-1+\Omega_{D}+\Omega_{D G P}+b^{2}\left(1-\Omega_{D G P}\right)\right)}{-2+\Omega_{D G P}+\Omega_{D}},
$$




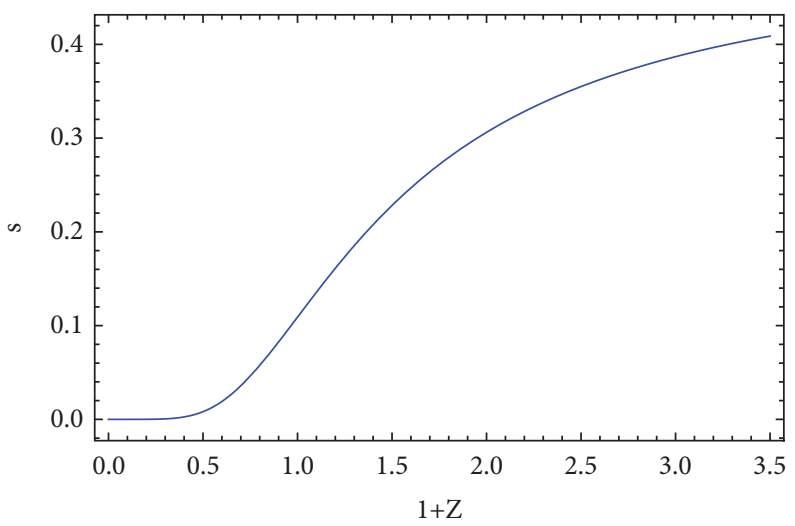

FIGURE 6: The evolution of the statefinder parameter $s$ versus the redshift parameter $z$ for noninteracting GDE in DGP model. Here, we have taken $\Omega_{D}(z=0)=0.73, H(z=0)=67$, and $\Omega_{r_{c}}=0.0003$.

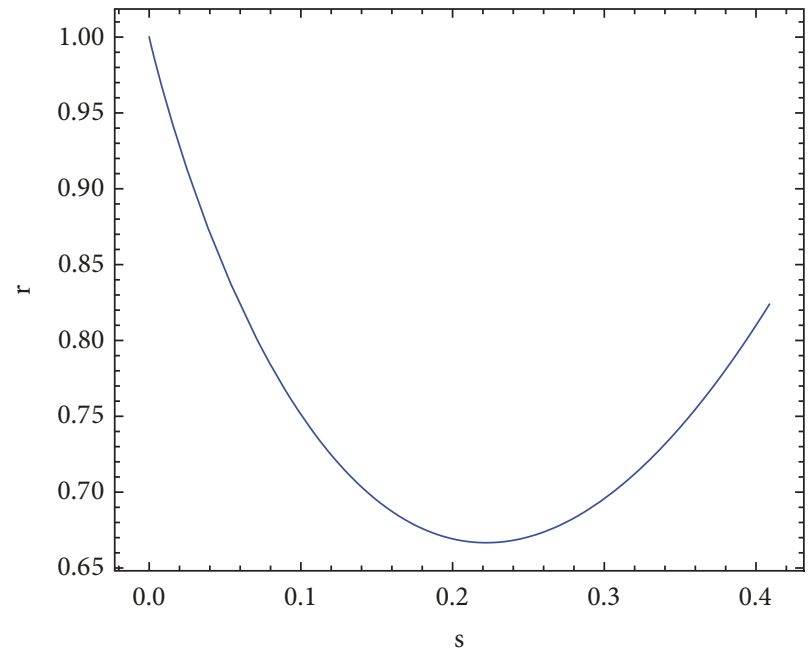

FIGURE 7: The evolution of the statefinder parameter $r$ versus $s$ for noninteracting GDE in the DGP model. Here, we have taken $\Omega_{D}(z=0)=$ $0.73, H(z=0)=67$, and $\Omega_{r_{c}}=0.0003$.

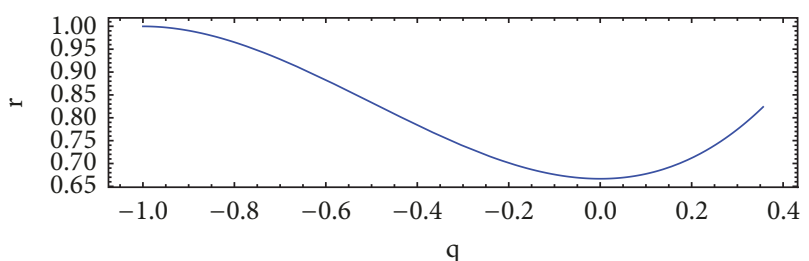

FIgURE 8: The evolution of the statefinder parameter $r$ versus the deceleration parameter $q$ for noninteracting GDE in the DGP model. Here, we have taken $\Omega_{D}(z=0)=0.73, H(z=0)=67$, and $\Omega_{r_{c}}=0.0003$.
To illustrate the cosmological consequences of the interacting GDE in the DGP braneworld, we plot their evolution in terms of redshift parameter $z$. In Figure 10, we present the graphical of $\Omega_{D}$ versus $z$ for the different values of the coupling constant $b^{2}$. As expected, we see both $\Omega_{D} \longrightarrow 1$ and $\Omega_{D} \longrightarrow 0$ for late time and early time, respectively. The graphical behavior of the EoS parameter for the different values of $b^{2}$ shows crossing of phantom line as plotted in Figure 11. The stability of interacting GDE in DGP model can be obtained by differentiating with respect to time of (23) and (29)

$$
v_{s}^{2}=-\frac{b^{2}\left[\left(-2+\Omega_{D G P}\right)^{3}+\Omega_{D}\left(6+\left(-6+\Omega_{D G P}\right) \Omega_{D G P}\right)\right]+\Omega_{D}\left(-2+2 \Omega_{D G P}+2 \Omega_{D}\right)}{\left(-2+\Omega_{D G P}\right)\left(-2+\Omega_{D G P}+\Omega_{D}\right)^{2}} .
$$

The evolution of the deceleration parameter $q$ and the squared of sound speed $v_{s}^{2}$ versus redshift parameter $z$ are plotted in Figures 12 and 13, respectively. In Figure 12, we see for different values of $b^{2}$ with the interacting GDE in DGP 


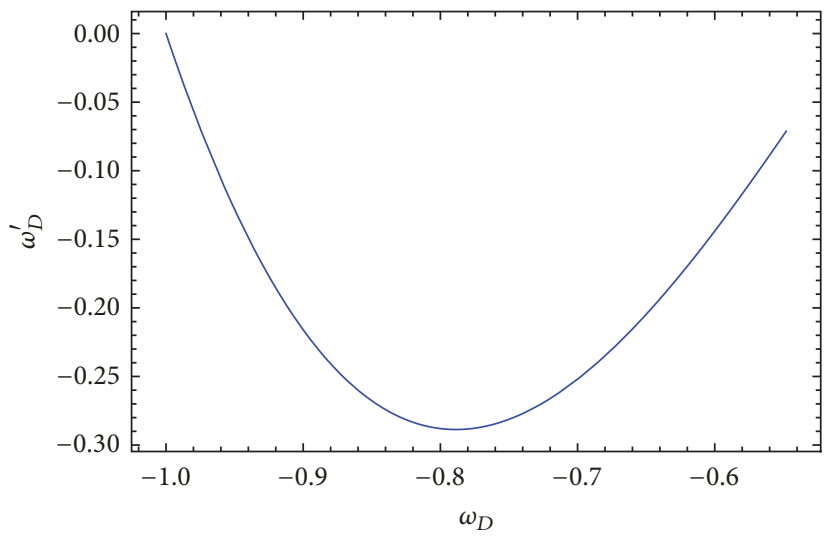

FIgURE 9: The $\omega_{D}-\omega_{D}^{\prime}$ diagram for noninteracting GDE in the DGP model. Here, we have taken $\Omega_{D}(z=0)=0.73, H(z=0)=67$, and $\Omega_{r_{c}}=0.0003$.

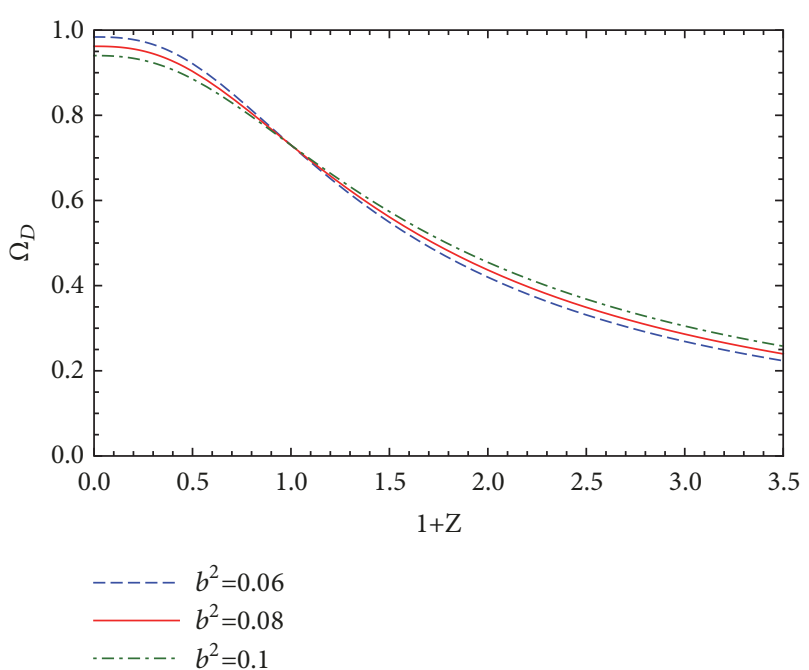

FIGURE 10: The evolution of $\Omega_{D}$ versus redshift parameter $z$ for interacting GDE in the DGP model. Here, we have taken $\Omega_{D}(z=$ $0)=0.73, H(z=0)=67$, and $\Omega_{r_{c}}=0.0003$.

model, our universe has a phase transition from deceleration to an acceleration, while by keeping the same situation in Figure 13, this universe cannot be stable. As the value of $b^{2}$ decreases the severity of instability also decreases. Like previous section, the statefinder parameters are obtained by taking derivative of (28) and using (13) and (15)

$$
\begin{aligned}
r= & 10+\frac{18\left(1+b^{2}-b^{2} \Omega_{D G P}\right)}{\left(-2+\Omega_{D G P}+\Omega_{D}\right)^{3}} \\
& +\frac{9\left(-1+b^{2}\left(-1+\Omega_{D G P}\right)\right)\left(-5+b^{2}\left(-3+2 \Omega_{D G P}\right)\right)}{\left(-2+\Omega_{D G P}+\Omega_{D}\right)^{2}} \\
& +\frac{9\left(4+b^{2}\left(4-3 \Omega_{D G P}\right)\right)}{-2+\Omega_{D G P}+\Omega_{D}},
\end{aligned}
$$

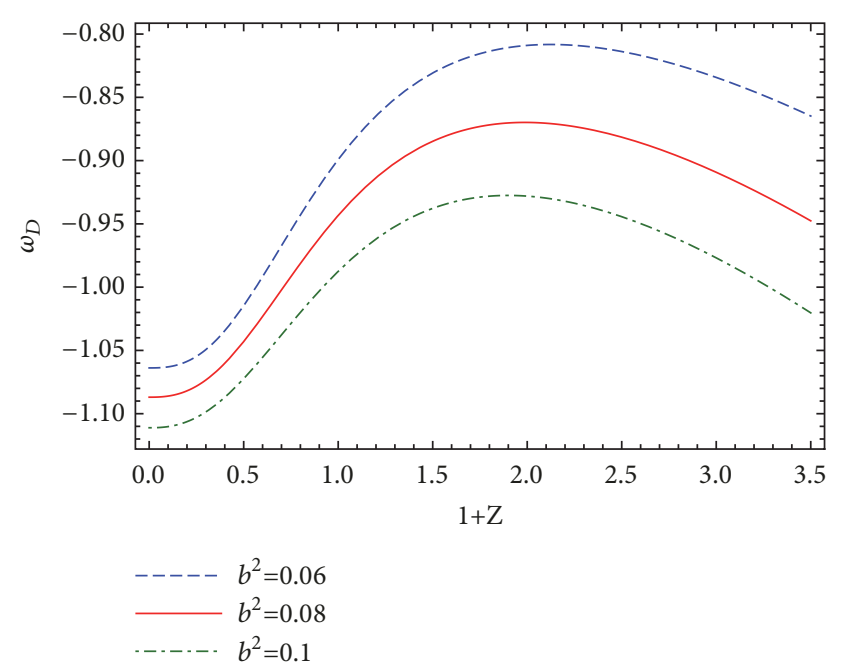

FIGURE 11: The evolution of $\omega_{D}$ versus redshift parameter $z$ for interacting GDE in the DGP model. Here, we have taken $\Omega_{D}(z=$ $0)=0.73, H(z=0)=67$, and $\Omega_{r_{c}}=.0003$.

$$
\begin{aligned}
s= & 2+\frac{2-2 b^{2}\left(-1+\Omega_{D G P}\right)}{\left(-2+\Omega_{D G P}+\Omega_{D}\right)^{2}}+\frac{4+b^{2}\left(3-2 \Omega_{D G P}\right)}{-2+\Omega_{D G P}+\Omega_{D}} \\
& +\frac{b^{2}}{2 b^{2}\left(1-\Omega_{D G P}\right)+\Omega_{D G P}+\Omega_{D}} .
\end{aligned}
$$

We obtain $\{r, s\}=\{1,0\}$ for $\Lambda$ CDM model from (33) and (34) in the limiting case where $b^{2}=0, \Omega_{D G P}=0$, and $\Omega_{D} \longrightarrow 1$ (in the late time). Also, Figures 14 and 15 show that $r$ and $s$ are positive through the entire life of the universe and turn to 1 and 0 at the late time, respectively. The $\{r, s\}$ evolutionary trajectories for the interacting GDE in the framework of the DGP braneworld for different values of $b^{2}$ are shown in Figure 16. From Figure 16, we can see that at the late time all curves tend to the $\Lambda \mathrm{CDM}$ fixed point $\{r=1, s=$ $0\}$; also different $b^{2}$ results in different evolution trajectories of statefinder which states that $r$ is smaller when $b^{2}$ is larger. The $r-q$ diagrams are plotted for different values of $b^{2}$ in Figure 17 


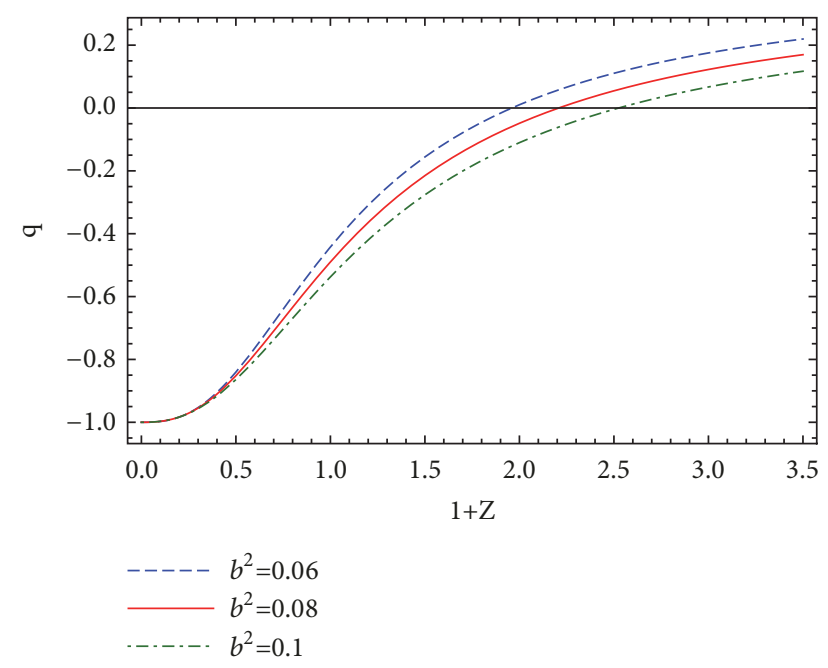

FIGURE 12: The evolution of the deceleration parameter $q$ versus redshift parameter $z$ for interacting GDE in the DGP model. Here, we have taken $\Omega_{D}(z=0)=0.73, H(z=0)=67$, and $\Omega_{r_{c}}=0.0003$.

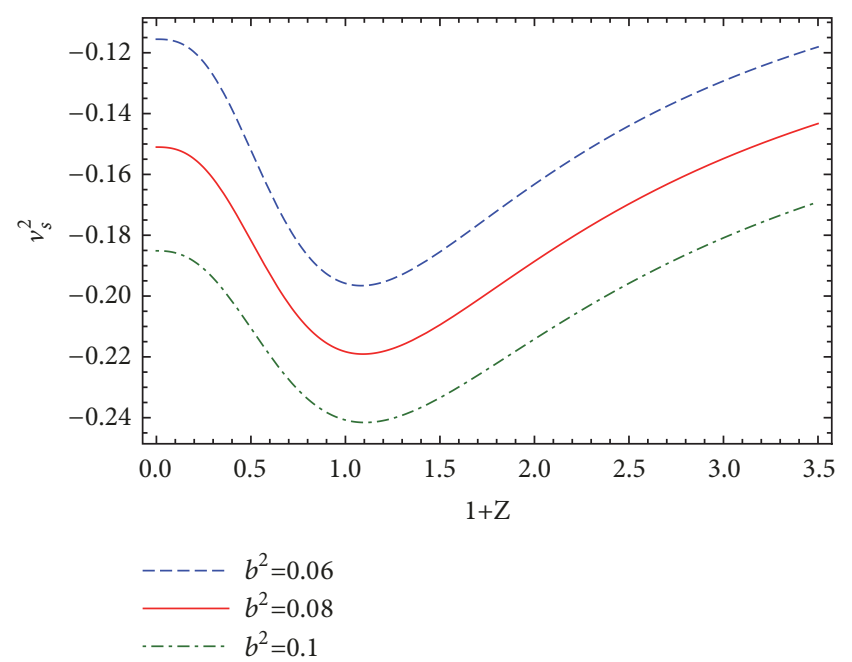

Figure 13: The evolution of the squared of sound speed $v_{s}^{2}$ versus redshift parameter $z$ for interacting GDE in the DGP model. Here, we have taken $\Omega_{D}(z=0)=0.73, H(z=0)=67$, and $\Omega_{r_{c}}=0.0003$.

which mimics the de Sitter expansion, namely, $r=1, q=-1$ in the far future where $z \longrightarrow 0$. In Figure 18, we plot the $\omega_{D}-\omega_{D}^{\prime}$ plane for different values of $b^{2}$ which show that the trajectories meet both the thawing and the freezing regions as well.

\section{Closing Remarks}

We have made a versatile study on both noninteracting and interacting GDE in the framework DGP model through well-known cosmological parameters as well as planes. We summarize our results as follows. For noninteracting case, we have found that the density parameter tends to zero at the early universe while at the late time we have $\Omega_{D} \longrightarrow$ 1. Meanwhile the EoS parameter cannot cross the phantom

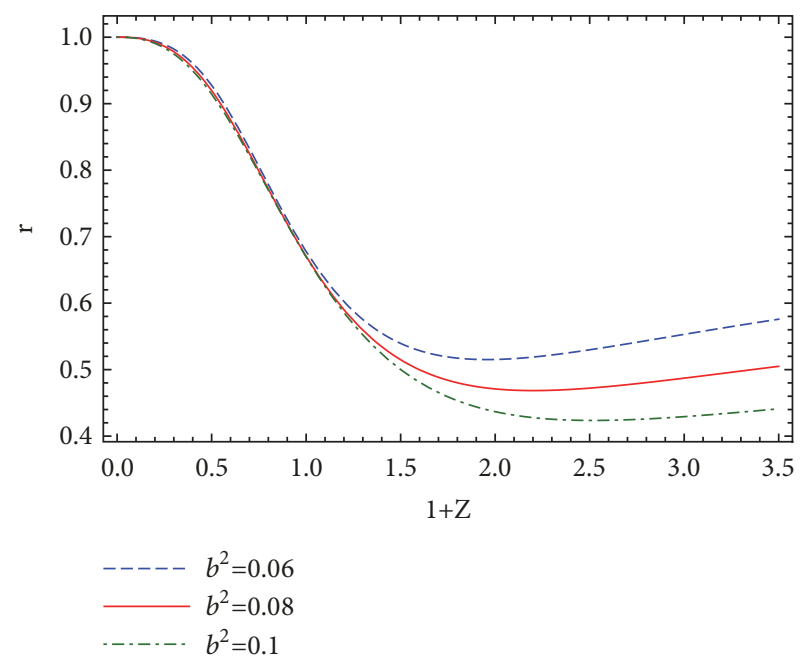

FIGURE 14: The evolution of the statefinder parameter $r$ versus the redshift parameter $z$ for interacting GDE in DGP model. Here, we have taken $\Omega_{D}(z=0)=0.73, H(z=0)=67$, and $\Omega_{r_{c}}=.0003$.

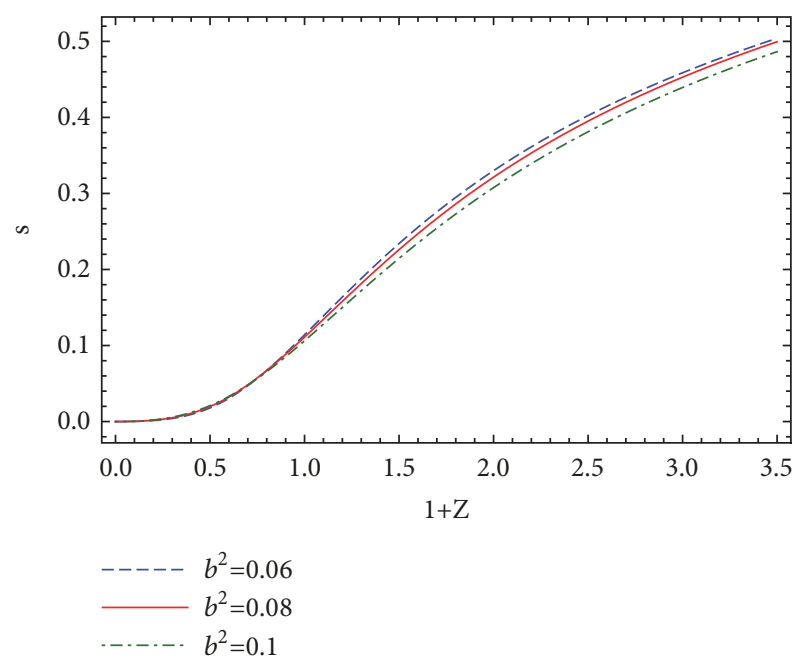

FIGURE 15: The evolution of the statefinder parameter $s$ versus the redshift parameter $z$ for interacting GDE in the DGP model. Here, we have taken $\Omega_{D}(z=0)=0.73, H(z=0)=67$, and $\Omega_{r_{c}}=.0003$.

line and mimics the cosmological constant at the late time (Figure 2). We have shown that our universe has a phase transition from deceleration to an acceleration, though we do not receive any signal of stability. The statefinder plane shows that the trajectory corresponds to quintessence model $(s>0$ and $r<1$ ) while at late time we have $\{r, s\}=\{1,0\}$ for $\Lambda$ CDM model as expected. The $\omega_{D}-\omega_{D}^{\prime}$ plane in Figure 9 meets the freezing region as well.

For interacting case, we find that the density and the deceleration parameters as well as the EoS parameter are consistent with observational data. We have seem that as the value of $b^{2}$ decreases the severity of instability decreases. From $r-s$ plane, we can see that at the late time all cures tend to the $\Lambda$ CDM fixed point $\{r=1, s=0\}$. Besides, for different values of $b^{2}$, the different evolution trajectories of statefinder 


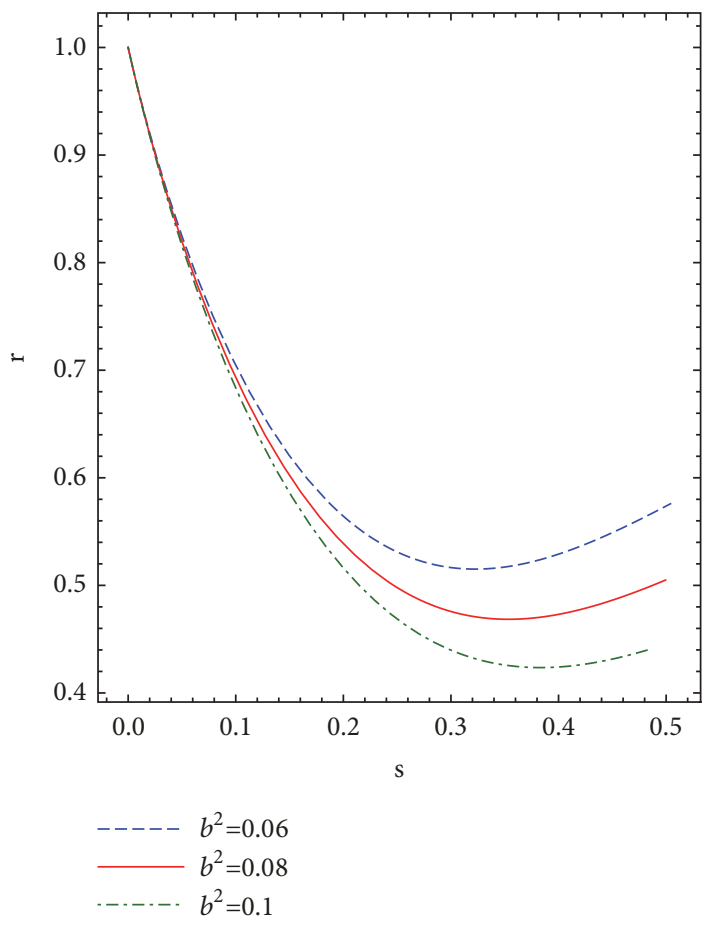

FIGURE 16: The evolution of the statefinder parameter $r$ versus $s$ for interacting GDE in the DGP model. Here, we have taken $\Omega_{D}(z=$ $0)=0.73, H(z=0)=67$, and $\Omega_{r_{c}}=.0003$.

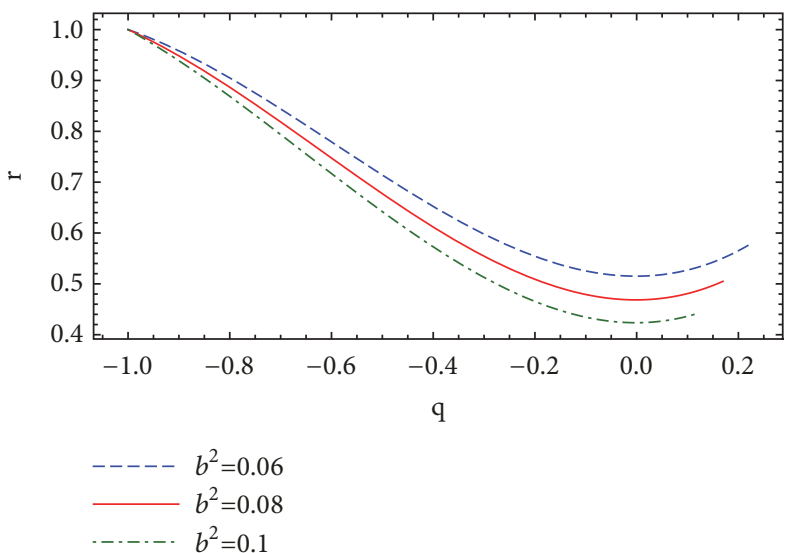

FIGURE 17: The evolution of the statefinder parameter $r$ versus the deceleration parameter $q$ for interacting GDE in the DGP model. Here, we have taken $\Omega_{D}(z=0)=0.73, H(z=0)=67$, and $\Omega_{r_{c}}=$ .0003 .

are shown which indicates that $r$ is smaller when $b^{2}$ is larger. The $r-q$ plane is plotted in Figure 17 which mimics the de Sitter expansion, namely, $r=1, q=-1$ in the far future where $z \longrightarrow 0$. In the end, the $\omega_{D}-\omega_{D}^{\prime}$ plane exhibits both freezing and thawing regions of the universe for all values of $b^{2}$. Again, in this case $v_{s}^{2}<0$ which implies that interacting GDE in the DGP braneworld is not stable against perturbation.

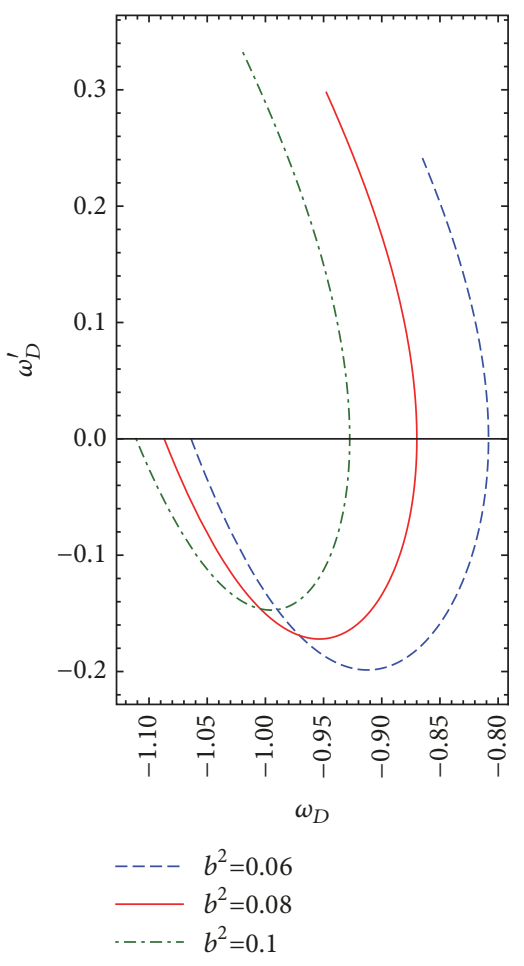

FIgURE 18: The $\omega_{D}-\omega_{D}^{\prime}$ diagram for interacting GDE in the DGP model. Here, we have taken $\Omega_{D}(z=0)=0.73, H(z=0)=67$, and $\Omega_{r_{c}}=.0003$.

\section{Data Availability}

No data were used to support this study.

\section{Conflicts of Interest}

The authors declare that they have no conflicts of interest.

\section{Acknowledgments}

The authors thank Shiraz University Research Council. This work has been supported financially by Research Institute for Astronomy \& Astrophysics of Maragha (RIAAM), Iran.

\section{References}

[1] S. Perlmutter, G. Aldering, and G. Goldhaber, "Measurements of $\Omega$ and $\Lambda$ from 42 High-Redshift Supernovae," The Astrophysical Journal, vol. 517, no. 2, pp. 565-586, 1999.

[2] A. G. Riess, A. V. Filippenko, P. Challis et al., "Observational evidence from supernovae for an accelerating universe and a cosmological constant," The Astronomical Journal, vol. 116, no. 3, pp. 1009-1038, 1998.

[3] G. Hinshaw, D. Larson, and E. Komatsu, "Nine-year Wilkinson Microwave Anisotropy Probe (WMAP) observations: cosmological parameter results," The Astrophysical Journal Supplement Series, vol. 208, no. 2, p. 19, 2013.

[4] S. Capozziello, V. F. Cardone, S. Carloni, and A. Troisi, "Curvature quintessence matched with observational data," 
International Journal of Modern Physics D, vol. 12, no. 10, pp. 1969-1982, 2003.

[5] S. Nojiri and S. D. Odintsov, "Introduction to modified gravity and gravitational alternative for dark energy," International Journal of Geometric Methods in Modern Physics, vol. 4, no. 1, article 115, 2007.

[6] S. Nojiri and S. D. Odintsov, "Unified cosmic history in modified gravity: from $\mathrm{F}(\mathrm{R}) \mathrm{F}(\mathrm{R})$ theory to Lorentz non-invariant models," Physics Reports, vol. 505, pp. 59-144, 2011.

[7] G. Papagiannopoulos, S. Basilakos, J. D. Barrow, and A. Paliathanasis, "New integrable models and analytical solutions in $\mathrm{f}(\mathrm{R})$ cosmology with an ideal gas," Physical Review D: Particles, Fields, Gravitation and Cosmology, vol. 97, no. 2, 2018.

[8] L. Amendola, "Scaling solutions in general nonminimal coupling theories," Physical Review D: Particles, Fields, Gravitation and Cosmology, vol. 60, Article ID 043501, 1999.

[9] J. Uzan, "Cosmological scaling solutions of nonminimally coupled scalar fields," Physical Review D: Particles, Fields, Gravitation and Cosmology, vol. 59, no. 12, 1999.

[10] A. Sheykhi, "Interacting new agegraphic dark energy in nonflat Brans-Dicke cosmology," Physical Review D: Particles, Fields, Gravitation and Cosmology, vol. 81, no. 2, 2010.

[11] K. Karami, A. Sheykhi, M. Jamil, Z. Azarmi, and M. M. Soltanzadeh, "Interacting entropy-corrected new agegraphic dark energy in Brans-Dicke cosmology," General Relativity and Gravitation, vol. 43, no. 1, pp. 27-39, 2011.

[12] A. Sheykhi, E. Ebrahimi, and Y. Yousefi, "Generalized ghost dark energy in Brans-Dicke theory," Canadian Journal of Physics, vol. 91, no. 8, pp. 662-667, 2013.

[13] F. Felegary, F. Darabi, and M. R. Setare, "Interacting holographic dark energy model in Brans-Dicke cosmology and coincidence problem," International Journal of Modern Physics D: Gravitation, Astrophysics, Cosmology, vol. 27, no. 3, 1850017, 18 pages, 2018.

[14] V. Sahni and A. Starobinsky, "The case for a positive cosmological $\Lambda$-term," International Journal of Modern Physics D, vol. 9, no. 4, p. 373, 2000.

[15] M. Li, "A model of holographic dark energy," Physics Letters B, vol. 603, no. 1-2, pp. 1-5, 2004.

[16] D. Pavon and W. Zimdahl, "Holographic dark energy and cosmic coincidence," Physics Letters B, vol. 628, no. 3-4, pp. 206210, 2005.

[17] M. R. Setare and E. N. Saridakis, "Non-minimally coupled canonical, phantom and quintom models of holographic dark energy," Physics Letters B, vol. 671, no. 3, pp. 331-338, 2009.

[18] A. Sheykhi, "Thermodynamics of interacting holographic dark energy with the apparent horizon as an IR cutoff," Classical and Quantum Gravity, vol. 27, no. 2, Article ID 025007, 2010.

[19] S. Nojiri and S. D. Odintsov, "Unifying phantom inflation with late-time acceleration: scalar phantom-non-phantom transition model and generalized holographic dark energy," General Relativity and Gravitation, vol. 38, pp. 1285-1304, 2006.

[20] R.-G. Cai, "A dark energy model characterized by the age of the universe," Physics Letters B, vol. 657, no. 4-5, pp. 228-231, 2007.

[21] A. Sheykhi, "Interacting agegraphic tachyon model of dark energy," Physics Letters B, vol. 682, no. 4-5, pp. 329-333, 2010.

[22] A. A. Mamon, "Reconstruction of interaction rate in holographic dark energy model with Hubble horizon as the infrared cut-off," International Journal of Modern Physics D, vol. 26, no. 11, 2017.
[23] M. A. Zadeh, A. Sheykhi, and H. Moradpour, "Holographic dark energy with the sign-changeable interaction term," International Journal of Modern Physics D: Gravitation, Astrophysics, Cosmology, vol. 26, no. 8, 1750080, 13 pages, 2017.

[24] B. Feng, X. L. Wang, and X. M. Zhang, "Dark energy constraints from the cosmic age and supernova," Physics Letters B, vol. 607, no. 1-2, pp. 35-41, 2005. 

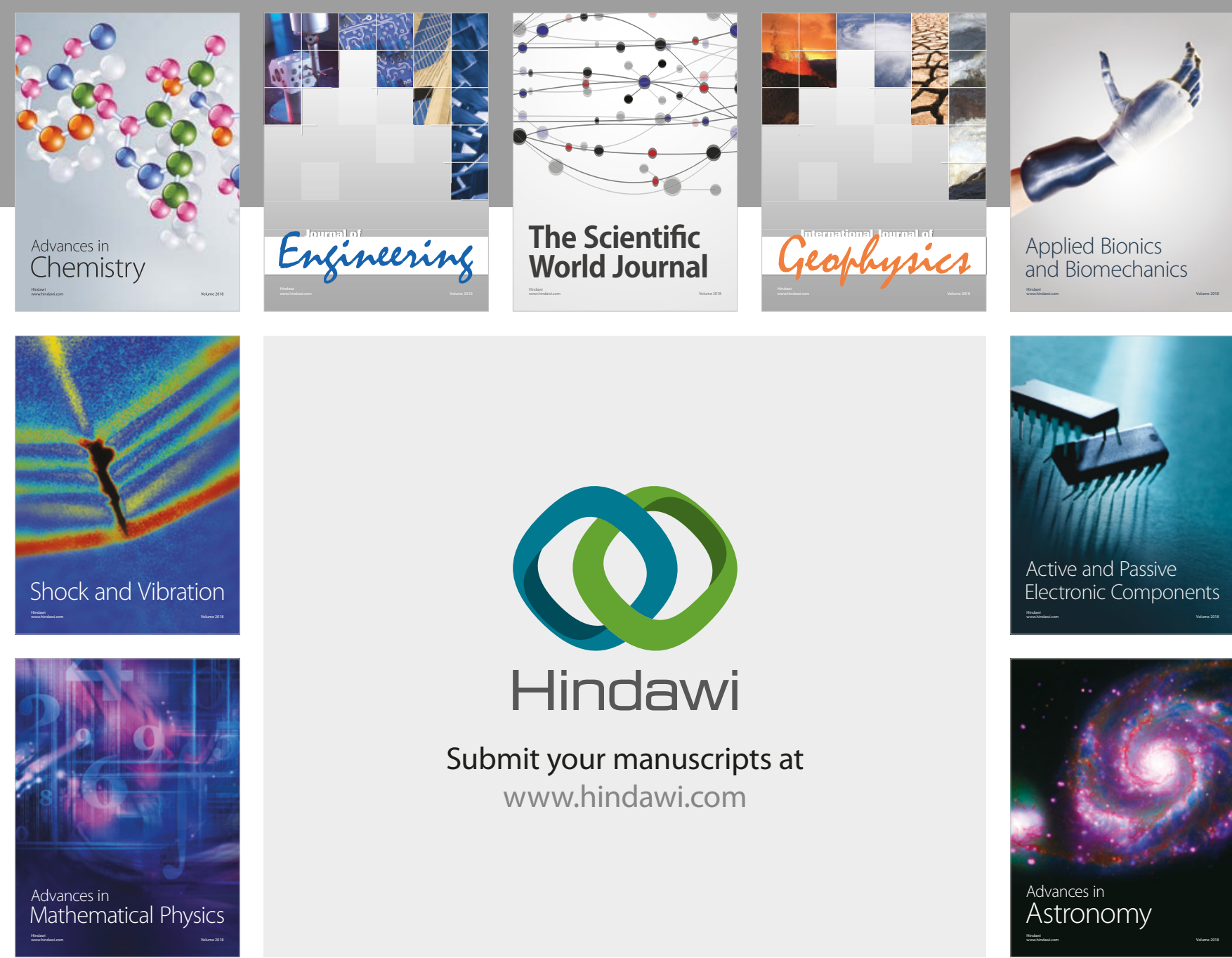

Submit your manuscripts at

www.hindawi.com

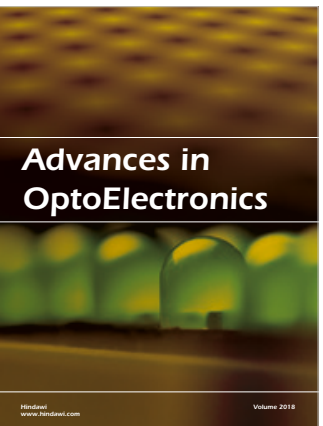

\section{Rotcting Machinery}
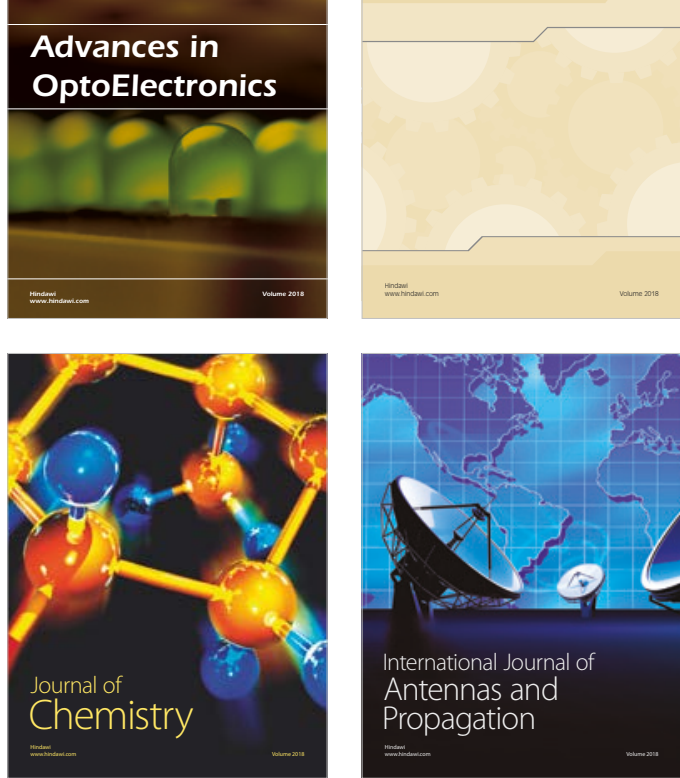

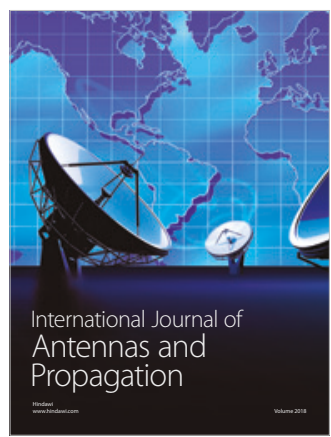

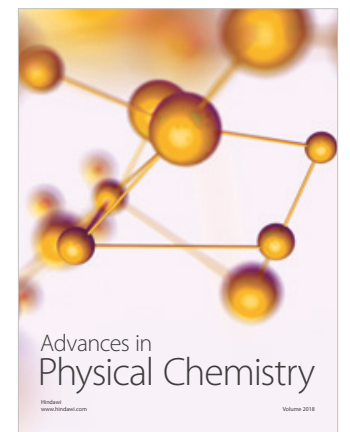

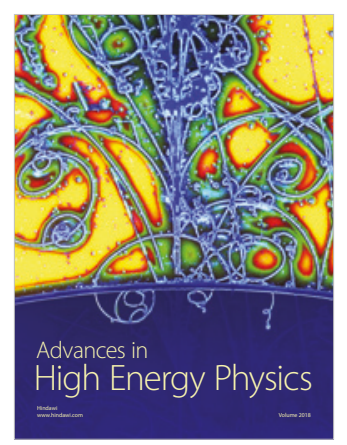

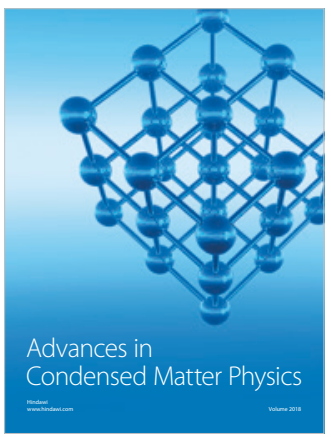

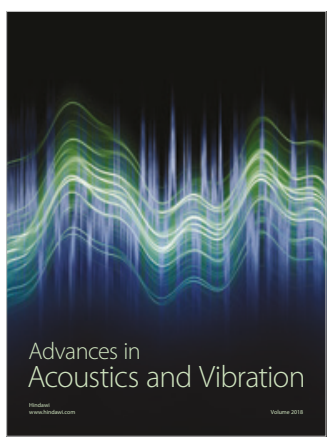

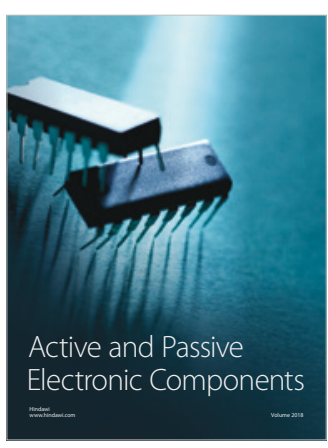
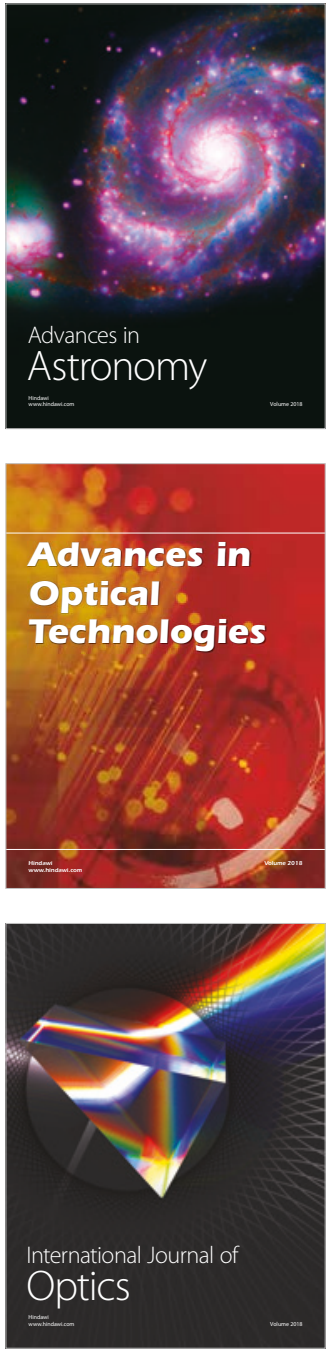